"NOTICE: this is the author's version of a work that was accepted for publication in Annals of Biomedical Engineering. Changes resulting from the publishing process, such as peer review, editing, corrections, structural formatting, and other quality control mechanisms may not be reflected in this document. Changes may have been made to this work since it was submitted for publication. A definitive version will be subsequently published in Annals of Biomedical Engineering, [VOL.37, pp. 1546-59, (2009)].”

\title{
MEASUREMENT OF INDIVIDUAL RED BLOOD CELL MOTIONS UNDER HIGH HEMATOCRIT CONDITIONS USING A CONFOCAL MICRO-PTV SYSTEM
}

\author{
Rui LIMA ${ }^{1,2}$, Takuji ISHIKAWA ${ }^{1}$, Yohsuke IMAI ${ }^{1}$, Motohiro TAKEDA ${ }^{1,3}$, Shigeo WADA $^{4}$, \\ Takami YAMAGUCHI
}

${ }^{1}$ Department of Bioengineering and Robotics, Graduate School of Engineering, Tohoku University, 66-01 Aoba, 980-8579 Sendai, Japan

${ }^{2}$ Department of Mechanical Tech, ESTiG, Braganca Polyt., C. Sta. Apolonia, 5301-857 Braganca, Portugal

${ }^{3}$ Division of Surgical Oncology, Graduate School of Medicine, Tohoku University, 2-1 Seiryo-machi, Aoba-ku, 980-8575 Sendai, Japan

${ }^{4}$ Department of Mechanical Science and Bioengineering, Graduate School of Engineering Science., Osaka University, Toyonaka, 560-8531 Osaka, Japan

${ }^{5}$ Department of Biomedical Eng., Grad. Biomedical Sch. Eng., Tohoku Univ., 6-6-01 Aoba, 980-8579

Sendai, Japan.

E-mail: ruimec@ipb.pt

Key words: Blood flow, Microcirculation, Red Blood Cells, Confocal micro-PTV, RBC dispersion, microchannels.

\begin{abstract}
Developments in optical experimental techniques have helped in elucidating how blood flows through microvessels. Although initial developments were encouraging, studies on the flow properties of blood in microcirculation have been limited by several technical factors, such as poor spatial resolution and difficulty obtaining quantitative detailed measurements at such small scales. Recent advances in computing, microscopy, and digital image processing techniques have made it possible to combine a particle tracking velocimetry (PTV) system with a confocal microscope. We document the development of a confocal micro-PTV measurement system for capturing the dynamic flow behavior of red blood cells (RBCs) in concentrated suspensions. Measurements were performed at several depths through $100-\mu \mathrm{m}$ glass capillaries. The confocal micro-PTV system was able to detect both translational and rotational motions of individual RBCs flowing in concentrated suspensions. Our results provide evidence that RBCs in dilute suspensions (3\% hematocrit) tended to follow approximately linear trajectories, whereas RBCs in concentrated suspensions (20\% hematocrit) exhibited transversal displacements of about $2 \%$ from the original path. Direct and quantitative measurements indicated that the plasma layer appeared to enhance the fluctuations in RBC trajectories owing to decreased obstruction in transversal movements caused by other RBCs. Using optical sectioning and subsequent image contrast and resolution enhancement, the system provides previously unobtainable information on the motion of RBCs, including the trajectories of two or more RBCs interacting in the same focal plane and RBC dispersion coefficients in different focal planes.
\end{abstract}




\section{INTRODUCTION}

Blood flow in the microcirculation depends on several combined effects, including cell deformability, flow shear rates, and vessel wall deformability, as well as biochemical and physiological factors. One of the first observations of the non-Newtonian behavior of blood flowing in capillaries was achieved by Fahraeus and Lindqvist ${ }^{1}$, who found that the apparent viscosity decreases with microtube diameter, in contrast to Poiseuille's law, for capillaries less than $300 \mu \mathrm{m}$ in diameter. However, explanations for this finding were only provided in the 1970 s through microscopic observations ${ }^{2-6}$. The most plausible explanation relies on the formation of a cell-free plasma layer near the wall, with red blood cells (RBCs) tending to migrate toward the center of the microtube. Extensive research ensued on the flow properties of blood in both dilute and concentrated suspensions using several measuring techniques such as double-slit photometry ${ }^{7,8}$, video microscopy and image analysis ${ }^{4-6,9}$, laser-Doppler anemometry $^{10,11}$, and particle-measuring methods ${ }^{12-15}$. Although extensive studies have been conducted on the microhemodynamic behavior of single RBCs in dilute suspensions, their behavior in concentrated suspensions remains poorly understood, in part due to technical limitations. The main limitation in measuring high concentrations is the attenuation of incident light owing to hemoglobin absorption and light scattering by the RBCs themselves. Thus, despite recent advances in microscopy, blood flow phenomena at a microscopic level, particularly in concentrated suspensions, remain unclear.

Results from Goldsmith and his colleagues (see Goldsmith et al. ${ }^{16}$ for review) regarding the flow behavior of RBCs in glass capillaries have provided the most insight into several microrheological phenomena occurring in highly concentrated RBC suspensions. However, their research employed transparent ghost cell suspensions to avoid light absorption by hemoglobin, which strongly influences the mechanical properties of RBC membranes. By removing hemoglobin, the membrane mechanical properties might have been altered appreciably, consequently changing the rheological behavior ${ }^{17-20}$. In addition, conventional microscopy illuminates the entire flow region, providing high background noise from out-of-focus emitted light and thereby degrading flow measurements ${ }^{21,22}$. Hence, more accurate measurements in concentrated $\mathrm{RBC}$ suspensions are required to gain further insight into complex microhemodynamic phenomena.

Recent progress in confocal microscopy ${ }^{22,23}$ have led to a new technique known as confocal microparticle image velocimetry (PIV) ${ }^{14,15,24-27}$. This method combines conventional PIV with a spinning disk confocal microscope (SDCM) to obtain in-focus images with an optical thickness of less than $1 \mu \mathrm{m}$ (optical sectioning effect). The superior spatial resolution and improved contrast and definition allow the measurement of several microrheology phenomena in concentrated suspensions of RBCs. Recently, our confocal system was combined with a cross-correlation technique, to obtain detailed information on the in vitro velocity profiles of blood with hematocrits (Hcts) up to $17 \%{ }^{14}$. However, velocity field errors resulting from trace particles necessitate combining the developed confocal system with a single particle tracking (SPT) method to characterize higher Hct level phenomena. To our knowledge, the present study provides the first confocal microparticle tracking velocimetry (PTV) measurements for studying microrheology in highly concentrated erythrocyte suspensions.

The present study demonstrates the ability of our confocal micro-PTV system to obtain detailed qualitative and quantitative information on the behavior of individual RBCs flowing through $100-\mu \mathrm{m}$ glass capillaries. Measurements were performed at $20 \mu \mathrm{m}$ from the bottom wall (20\% Hct; Reynolds number, 0.006$)$. We also measured radial displacement and dispersion coefficients at several depths and $\operatorname{Hcts}(3,15$, and $35 \%)$. 


\section{MATERIALS AND METHODS}

\section{Blood cell labeling}

We examined four working fluids: Dextran 40 (Dx-40; Otsuka Medicine, Tokyo, Japan) containing $3 \%(3 \% \mathrm{Hct}), 15 \%(15 \% \mathrm{Hct})$, or $35 \%(35 \% \mathrm{Hct})$ human RBCs, and Dx-40 containing $20 \%$ (20\% Hct) RBCs and 2\% white blood cells (WBCs). Both the RBCs and WBCs were labeled with a lipophilic carbocyanime derivative, chloromethylbenzamido (CM-Dil, C-7000, Molecular Probes, Eugene, OR, USA). This cell tracker was selected because of its strong photostable fluorescence, excellent cellular retention, and minimal cytotoxicity (see Fig. 1).
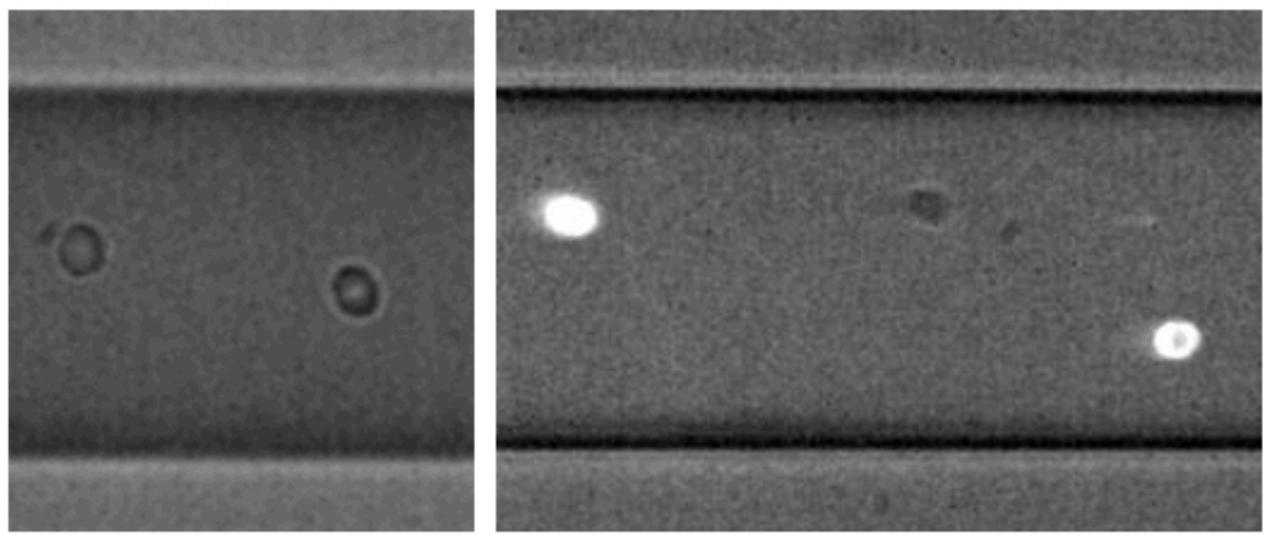

Figure 1. Halogen and confocal images of fluorescently labeled RBCs. The blood cells were labeled with a lipophilic carbocyanime derivative, chloromethylbenzamido (CM-Dil, C-7000). On the left side two RBCs appear as bright spots from laser-emitted light.

Blood was collected from a healthy adult volunteer (age, 33 years); ethylenediaminetetraacetic acid (EDTA) was used to prevent coagulation. RBCs were separated by centrifugation (1500 rpm, $20 \mathrm{~min}$ ), and the plasma and buffy coat were removed by aspiration; washing and centrifugation with physiological saline (PS) were repeated twice. The washed RBCs were diluted with PS to make several samples with hematocrit levels of $\sim 40 \%$ by volume. All blood samples were stored hermetically at $4^{\circ} \mathrm{C}$ until labeling.

The procedure for labeling both RBCs and WBCs was as follows:

1. Centrifuge the blood sample ( $1 \mathrm{~mL}$ with $40 \% \mathrm{Hct})$ at $2000 \mathrm{rpm}$ for $5 \mathrm{~min}$;

2. Remove all PS and add $200 \mu \mathrm{L}$ of fresh PS and $10 \mu \mathrm{L}$ of cell tracker CM-Dil, C-7000 (50 $\mu \mathrm{g}$, diluted with $200 \mu \mathrm{L}$ of ethanol);

3. Mix gently and incubate for $30 \mathrm{~min}$ at $37^{\circ} \mathrm{C}$, and then for $1-2 \mathrm{~h}$ at $4{ }^{\circ} \mathrm{C}$;

4. Add approximately $500 \mu \mathrm{L}$ of PS and mix gently;

5. Centrifuge the solution (2000 rpm, $5 \mathrm{~min}$ ) and remove the excess dye; and

6. Resuspend the washed cells in Dx-40 to the required $\mathrm{RBC}$ or $\mathrm{WBC}$ concentration by volume.

Owing to possible loss of blood cells during labeling, the hematocrit of each sample was measured using a hematocrit centrifuge (Kubota 3220; Kubota Corp., Osaka, Japan) prior to the experiment. Note that WBCs were obtained using a lymphoprep tube (Axis-Shield, Oslo, Norway) according to the separation procedure recommended by the manufacturer. Using this approach, fluorescently labeled RBCs comprised about $10 \%$ of the total cell volume ( $40 \% \mathrm{Hct})$.

All procedures were carried out in compliance with the guidelines of the Ethics Committee on Clinical Investigation of Tohoku University. 


\section{Glass microchannels}

A $100-\mu \mathrm{m}$, circular, borosilicate glass microchannel was fabricated by Vitrocom (Mountain Lakes, NJ, USA). The microchannel was mounted on a slide glass approximately $80 \mu \mathrm{m}$ thick and immersed in glycerol of the same refractive index to minimize refraction from the walls.

\section{Experimental setup}

The confocal micro-PTV system consisted of an inverted microscope (IX71; Olympus, Tokyo, Japan) combined with a confocal scanning unit (CSU22; Yokogawa, Tokyo, Japan) and a diode-pumped solid state (DPSS) laser (Laser Quantum Ltd., Stockport, UK), using an excitation wavelength of $532 \mathrm{~nm}$. A high-speed camera (Phantom v7.1; Vision Research, NJ, USA) was connected to the outlet port of the CSU22 (Fig. 2). The glass microtube was placed on the stage of the inverted microscope, where the flow rate of the working fluids was kept constant using a syringe pump (KD Scientific Inc., Holliston, MA, USA). The Reynolds number (Re) and associated experimental parameters are summarized in Table 1. Using a thermo-plate controller (Tokai Hit, Shizuoka, Japan), the temperature around the microtube was maintained at $36 \pm 1{ }^{\circ} \mathrm{C}$.

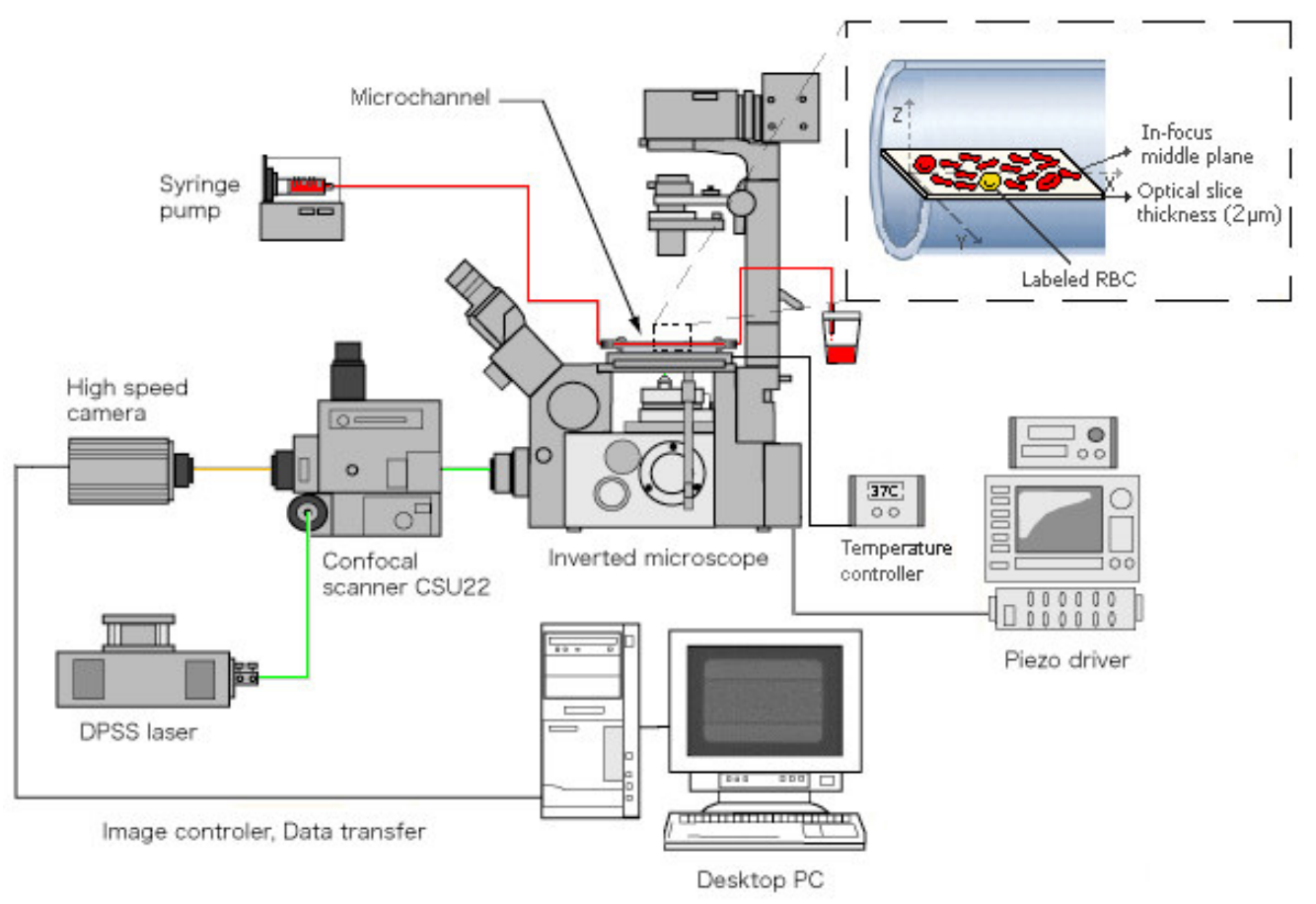

Figure 2. Confocal micro-PTV experimental setup. The confocal system consisted of an inverted microscope combined with a confocal scanning unit (CSU22), a DPSS laser and a high-speed camera.

Table 1. Experimental parameters used to calculate the Re.

\begin{tabular}{|c|c|c|c|c|c|c|c|}
\hline Experiment & $\mathrm{n}^{*}$ & Hct $(\%)$ & $\begin{array}{l}\text { Density } \\
\left(\mathrm{kg} / \mathrm{m}^{3}\right)\end{array}$ & $\begin{array}{c}\text { Mean } \\
\text { velocity } \\
(\mathrm{m} / \mathrm{s})\end{array}$ & $\begin{array}{l}\text { Diameter } \\
(\mathrm{m})\end{array}$ & $\begin{array}{c}\text { Viscosity of } \\
\text { Dx-40 } \\
\left(\mathrm{Ns} / \mathrm{m}^{2}\right) \\
\end{array}$ & $\mathrm{Re}$ \\
\hline $\begin{array}{l}\text { RBC and WBC flow near } \\
\text { the wall }(\mathrm{z}=20 \mu \mathrm{m})\end{array}$ & 51 & 20 & 1046 & $2.5 \times 10^{-4}$ & $10^{-4}$ & $4.5 \times 10^{-3}$ & 0.0058 \\
\hline \multirow{3}{*}{$\begin{array}{l}\text { RBC dispersion at a series } \\
\text { of optical sections }\end{array}$} & 74 & 3 & 1046 & $1.63 \times 10^{-4}$ & $10^{-4}$ & $4.5 \times 10^{-3}$ & 0.0038 \\
\hline & 92 & 15 & 1046 & $1.85 \times 10^{-4}$ & $10^{-4}$ & $4.5 \times 10^{-3}$ & 0.0043 \\
\hline & 46 & 35 & 1046 & $1.8 \times 10^{-4}$ & $10^{-4}$ & $4.5 \times 10^{-3}$ & 0.0042 \\
\hline
\end{tabular}

*Number of labeled RBCs. 
The laser illuminated the glass microchannel from beneath the microscope stage, through a dry $\times 40$ objective lens with a numerical aperture of 0.9. Satisfactory illumination of the labeled RBCs and WBCs, which absorbed green light (excitation peak, $553 \mathrm{~nm}$ ) and emitted yellow light (emission peak, $570 \mathrm{~nm}$ ), was achieved. The optical slice thickness was $2 \mu \mathrm{m}$, based on geometric-optical analysis criteria $^{26}$ (Fig. 3).

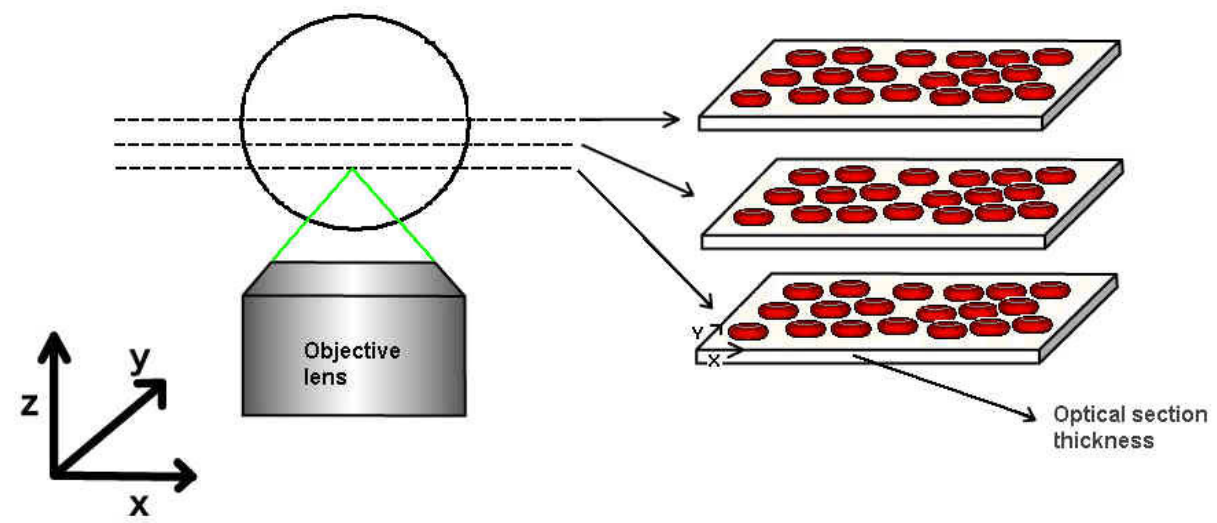

Figure 3. Diagram showing the ability of the confocal system to generate optical sectioned images along the $z$ axis. The optical slice thickness (OST) was $2 \mu \mathrm{m}$ based on geometric-optical analysis criteria ${ }^{26}$. Images were captured with a resolution of $640 \times 480$ pixels, at a rate of 100 frames/s with an exposure time of $9.4 \mathrm{~ms}$. The temporal resolution of the measurements was $10 \mathrm{~ms}$.

A series of $x y$ confocal images was captured in 12-bit gray scale, with a resolution of $640 \times 480$ pixels, and at a rate of $100 \mathrm{frames} / \mathrm{s}$ using an exposure time of $9.4 \mathrm{~ms}$, which corresponded to a temporal resolution of $10 \mathrm{~ms}$. The use of this long exposure time $(9.4 \mathrm{~ms})$ allowed us to improve the image brightness owing to an increase in the residence time of the labeled RBCs, which enabled the gathering of sufficient photons for image formation. As a result, it was possible to track labeled RBCs from one picture to the next. Recorded images were evaluated with Image $\mathrm{J}^{28}$ software (NIH, Bethesda, MD, USA), using the manual tracking MtrackJ ${ }^{29}$ plug-in.

\section{Tracking single RBCs: Trajectory, collision, and rolling}

Using the labeling procedure described above, most of the RBCs were uniformly labeled along the entire membrane surface (see Fig. 1). The fluorescently labeled RBCs lying in the focal plane of interest appeared as bright dots, with the highest emitted light intensity. To minimize the effects of the fainter RBCs passing through the region of interest but above and below the focal plane, a specific threshold intensity level was chosen. Thus, data for all labeled RBCs with intensities below 100 (8-bit grayscale; min-max, 0-255) were discarded. An exception was made when examining the interaction effects of WBCs on the motion of RBCs.

The RBCs were followed in flow for periods of time from 0.34 to $1 \mathrm{~s}$, with a temporal resolution of $10 \mathrm{~ms}$. To determine the motion of selected RBCs, they were manually tracked through successive images, using the intensity-weighted mean position of pixels above a threshold (bright centroid). With this method, it was possible to track labeled RBCs even when two cells were in close proximity. After obtaining a series of $x$ and $y$ positions, the data were exported for the determination of physical quantities such as the velocity, radial and transversal displacement, and dispersion coefficient, using proprietary $\mathrm{C}$ code software ${ }^{30}$.

The brightness of fluorescently labeled RBCs depended strongly on their location within the microchannel. During the experiments, especially at locations close to the bottom wall (strongest incident light), we found that a small percentage of RBCs (less than $1 \%$ of the total cell volume) were partially labeled, creating small localized markers along the membrane. This phenomenon allowed us 
to determine the membrane motion of the partially labeled RBCs flowing near the microchannel wall $(z=20 \mu \mathrm{m})$. Note that for this case, the tracking of the membrane motion was based on the bright centroid criteria, whereas the translational motion was based on the dark centroid criteria, using the MtrackJ ${ }^{29}$ software.

\section{RESULTS}

To analyze the ability of the confocal micro-PTV system to track RBCs, labeled RBCs at several Hcts (3-35\%) were followed. To calculate dispersion coefficients $\left(D_{y y}\right)$, measurements were performed at a series of optical sections. For complex microrheological events in flowing blood, such as RBC interaction and orientation, all measurements were performed near the microchannel wall $(z=20 \mu \mathrm{m})$ with $\sim 20 \%$ Hct. Wall region phenomena are of primary interest due to the lack of understanding surrounding mass transport mechanisms in thrombogenesis ${ }^{31,32}$.

\section{Blood flow visualization}

\section{$R B C$ and $W B C$ flow at $20 \% \mathrm{Hct}$}

The interactions of blood cells in flowing blood and their orientations were measured in vitro at $20 \%$ Hct and Re $\sim 0.006$. Examples of recorded images in both the center and bottom planes $(z=20 \mu \mathrm{m})$ are presented (Fig. 4). In Figure 4a, the plasma layer formed is clearly visible. In Figure $4 \mathrm{~b}$, it is possible to see both labeled and unlabeled RBCs. Figures $4 \mathrm{c}$ and $4 \mathrm{~d}$ are confocal images used to track blood cells. Note that in Figure 4d, the brightness and contrast of the image were adjusted to better visualize both RBCs and WBCs. For a video of in vitro blood ( $20 \% \mathrm{Hct}$; Re, 0.006$)$ flowing around the center plane, see Supplementary Video 1 online.

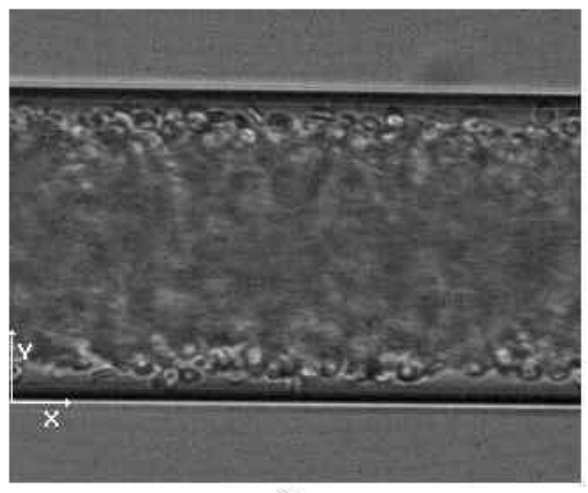

a)

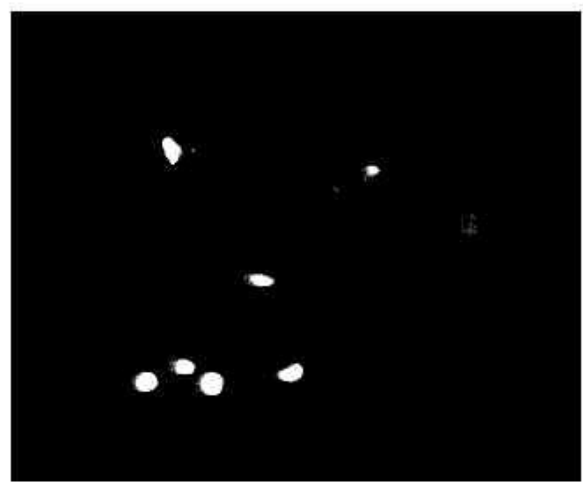

c)

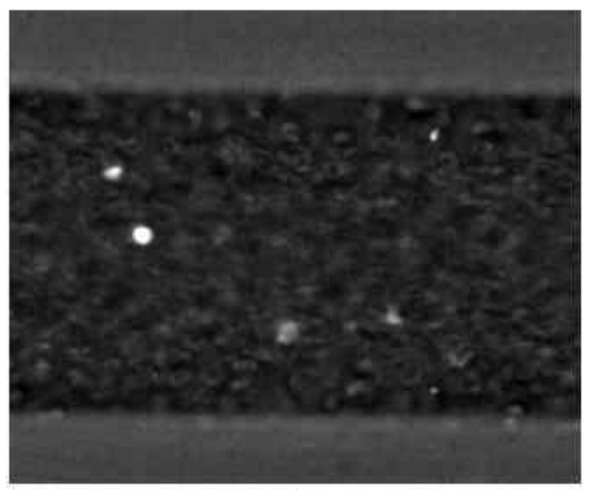

b)

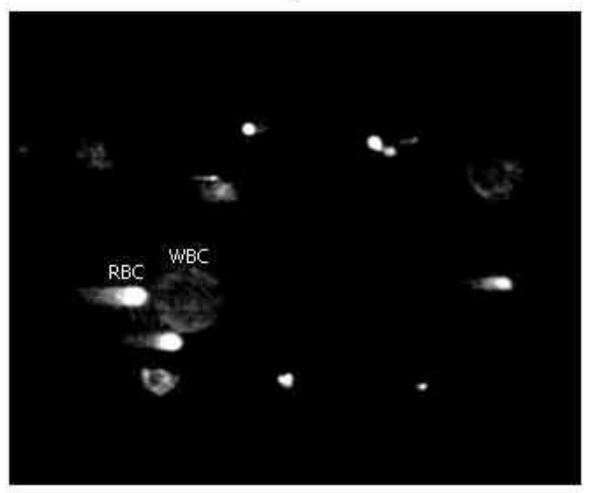

d)

Figure 4. a) An image of the blood used in this experiment with halogen illumination recorded in the center plane. b) An image with labeled RBCs (bright spots from laser-emitted light) and non-labeled RBCs (halogen illumination source) recorded in the bottom plane $(z=20 \mu \mathrm{m})$. c) A confocal image used to track RBC motion. d) A treated confocal image to visualize both RBCs and WBCs. 
RBC flow at several Hcts around the middle plane

$\mathrm{RBCs}$ in flowing blood exhibit a random-like, transverse motion, which is dependent on the Hct. Dispersion of labeled RBCs was measured in the center plane at several Hcts $(3,15,35 \%)$ at a $\operatorname{Re}$ of $\sim 0.004$. Figure 5 shows images recorded with both labeled and unlabeled RBCs at several Hcts. It is evident that the plasma layer tends to decrease with an increasing Hct.

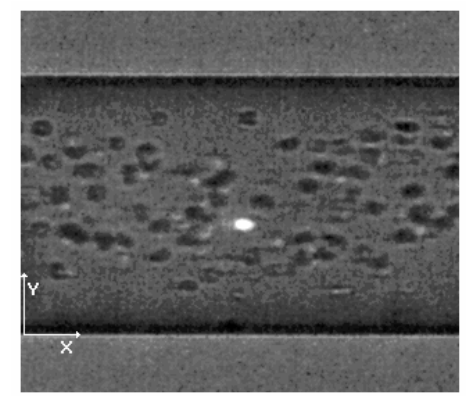

a) $3 \mathrm{Hct}$

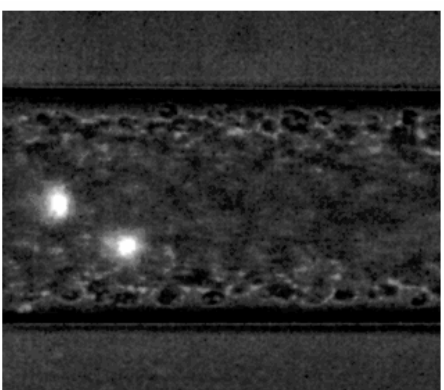

b) $15 \mathrm{Hct}$

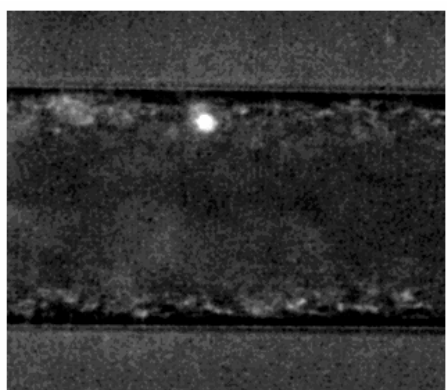

c) $35 \mathrm{Hct}$

Figure 5. Halogen and confocal images in the middle plane with (a) $3 \%$ Hct, (b) $15 \%$ Hct, and (c) $35 \%$ Hct. The non-labeled RBCs are observed as dark-gray rings, whereas labeled RBCs are observed as bright rings. It is possible to observe that the plasma layer tends to decrease with increasing Hct.

\section{Motion of individual RBCs}

In vitro blood $(20 \% \mathrm{Hct})$ was used to evaluate the ability of the proposed confocal system to investigate erythrocyte behavior in crowded environments $(\mathrm{Re}, \sim 0.006)$. We were only able to measure cell-cell interactions at $z=20 \mu \mathrm{m}$, because of the formation of a plasma layer close to the wall. The microrheology phenomena studied were RBC-RBC and RBC-WBC hemodynamic interactions, as well as RBC orientation with and without interaction. Several representative examples are presented below. In this particular study, the hemodynamic interaction was quantified by the transversal displacement, $R_{y}(t)$, which is the y position normal to the direction of the flow in a time interval, $t$.

\section{$R B C-R B C$ interactions in flowing blood}

The individual interaction measurements shown in the present study may help in further understanding the statistical values of the RBC dispersion coefficient obtained in a recent study performed by the current authors (Lima et al., 2008) ${ }^{37}$. In the present study, we showed the unique ability of the confocal system to obtain thin in-focus planes, allowing the measurement of the paths of two or more labeled RBCs interacting in the same focal plane. Although we were able to measure hundreds of individual $\mathrm{RBC}$ trajectories, measuring the interaction of two labeled RBCs was extremely complex. Two successful examples of RBC-RBC interaction within the plasma layer for a Re of $\sim 0.006$ are shown in Figures 6 and 7. These examples show the effect on the trajectories of two interacting RBCs around the crowded boundary region of the plasma layer (Fig. 6) and adjacent to the microtube wall (Fig. 7).

Figure 6 shows typical trajectories of two-RBC interactions around the crowded boundary region of the plasma layer, where the disturbance effect is increased by the collision of a neighboring RBC. Here, the transversal displacement $\left(R_{y}\right)$ of both RBCs increased about 2-3 $\mu \mathrm{m}$ due to their collision $(t=0.15 \mathrm{~s})$. For RBC 2, it is evident that the displacement continued to increase because of not only the current interaction but also other interactions with neighboring cells.

The trajectories of two RBCs interacting near the microtube wall are shown in Figure 7 . RBC 3 maintained a constant displacement along the wall (particularly for $t>0.24 \mathrm{~s}$ ), whereas $\mathrm{RBC} 4$ suffered an evident disturbance of its trajectory. These results suggest that the trajectories of RBCs rolling on the wall surface do not experience a strong hemodynamic effect from neighboring RBCs. Note that RBC 4 first undergoes a steep transversal displacement of about $3 \mu \mathrm{m}$, followed by a gradual decrease, probably as a result of collisions with the crowded suspension environment that exists adjacent to the plasma layer. 


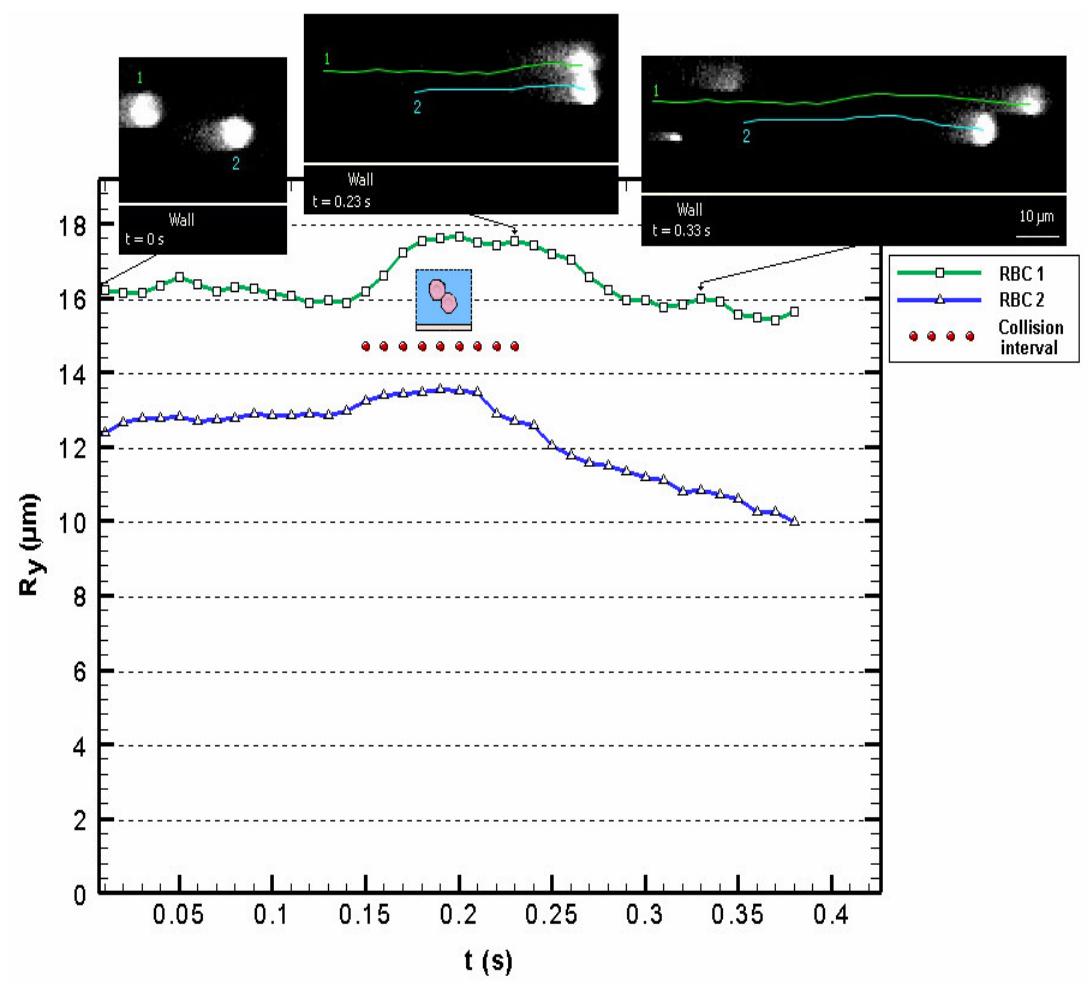

Figure 6. Two-RBC interactions at different times intervals $(\mathrm{Re} \sim 0.006)$. Instantaneous transversal displacement $\left(\mathrm{R}_{\mathrm{y}}\right)$ of two-RBC interactions at $10 \mathrm{~ms}$ time intervals tracked in the bottom plane $(z=20 \mu \mathrm{m})$ of a 100 $\mu \mathrm{m}$ diameter microchannel. See Supplementary Video 2.

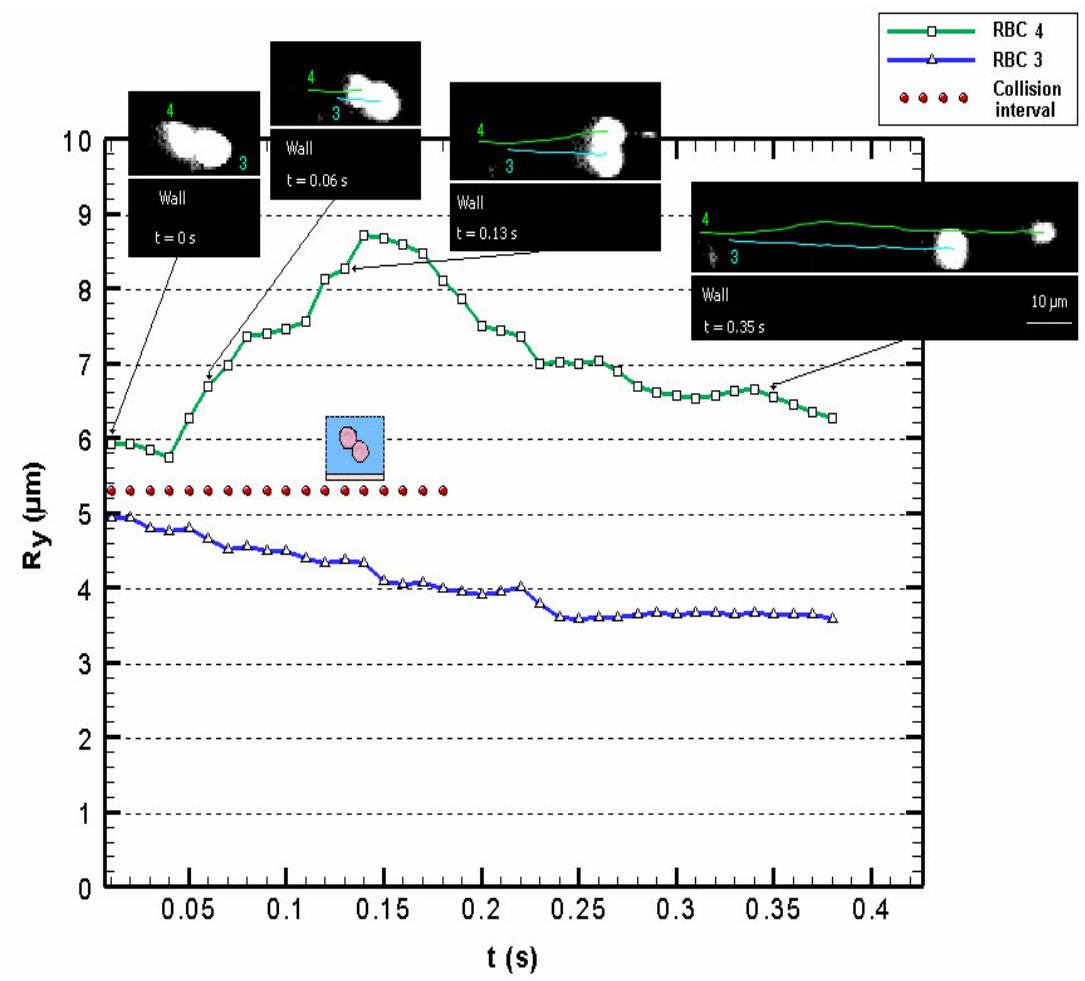

Figure 7. Two-RBC interactions near the wall ( $\mathrm{Re} \sim 0.006)$. Instantaneous transversal displacement $\left(\mathrm{R}_{\mathrm{y}}\right)$ of two$\mathrm{RBC}$ interactions at $10 \mathrm{~ms}$ time intervals tracked in the bottom plane $(z=20 \mu \mathrm{m})$ of a $100 \mu \mathrm{m}$ diameter microchannel. RBC6 collides with a slower RBC rolling on the wall of the microchannel. See Supplementary Video 3. 
Figure 8 illustrates the instantaneous transversal positions of two RBCs (RBC 4 and RBC 2) that interacted continuously with neighboring RBCs at a $20 \%$ Hct. Transversal displacements of two noninteracting RBCs (RBCnoInt1 and RBCnoInt2) are also shown for a Hct of 3\%. These results demonstrate that for the case of non-interacting RBCs, the magnitude of the transversal variability is always less than $1 \mu \mathrm{m}$, whereas RBCs flowing within a crowded environment experience greater variability in their trajectories, from $\sim 2$ up to $3 \mu \mathrm{m}$. Thus, the degree of convective dispersion is Hct dependent. These findings are consistent with those of our recent study on the determination of the RBC radial dispersion in a 100- $\mu \mathrm{m}$ microchannel at different Hcts (Lima et al., 2008) ${ }^{37}$.

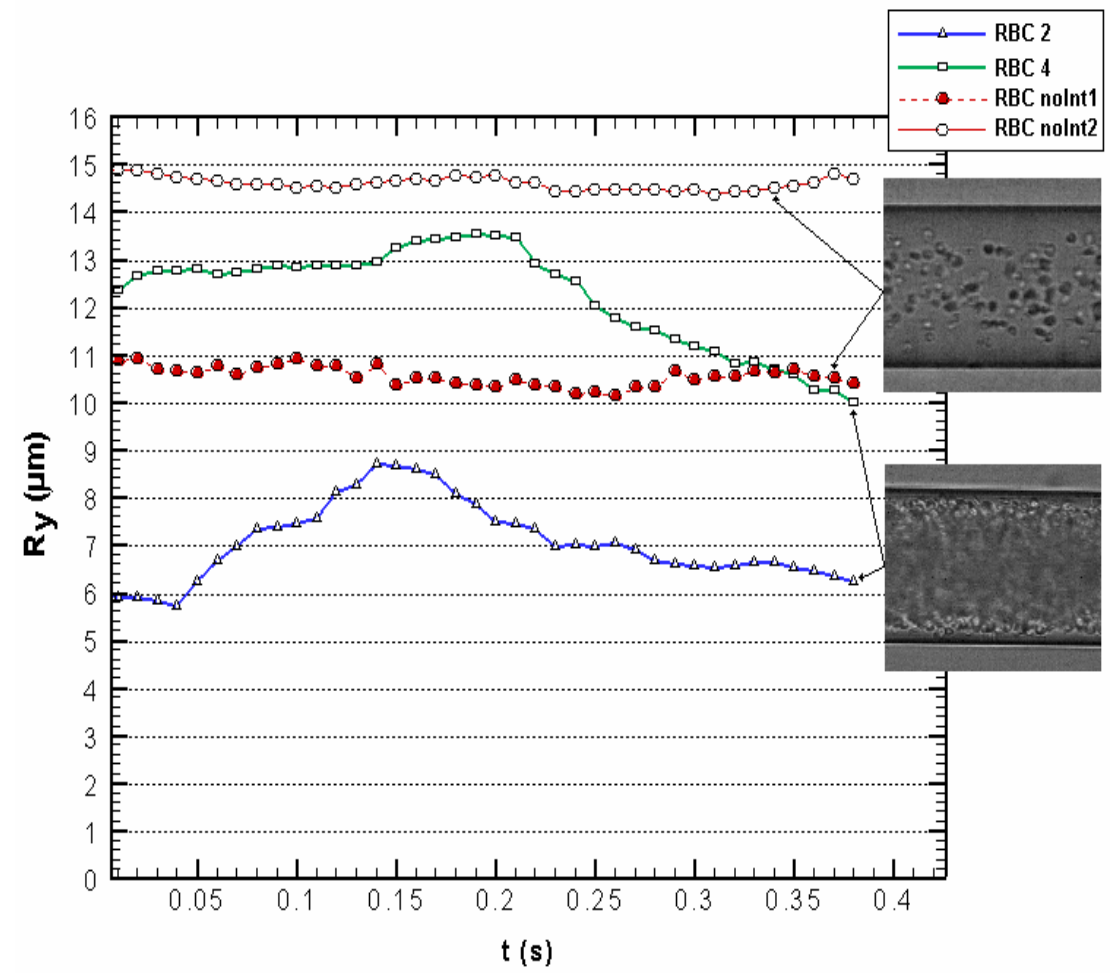

Figure 8. Comparison of the transversal displacement $\left(\mathrm{R}_{\mathrm{y}}\right)$ between $\mathrm{RBCs}$ with interactions $(20 \% \mathrm{Hct})$ and RBCs with no interactions at low Hct (3\% Hct). RBCS traveling in dilute suspensions (3\% Hct) tend to follow approximately linear trajectories whereas at concentrated suspensions $(20 \% \mathrm{Hct}) \mathrm{RBCs}$ suffer a variability in their trajectories of at least $1 \mu \mathrm{m}$ from its original path.

\section{Orientation of RBCs in flowing blood}

The orientations of two RBCs with and without interaction were measured around the plasma layer in concentrated suspensions. By adjusting the image contrast, it was possible to measure both translational and rotational motion. The translational motions were measured at center of the RBC (based on the dark centroid criteria), whereas rotation was measured along the membrane (based on the bright centroid criteria), using MtrackJ ${ }^{29}$ software (Fig. 9).

The brightness of fluorescently labeled RBCs depended strongly on their location within the microchannel. During the experiments, especially at locations close to the bottom wall (strongest incident light), we found that a small percentage of RBCs (less than $1 \%$ of the total cell volume) was partially labeled, creating small localized markers along the membrane. This phenomenon allowed us to determine the membrane motion of the partially labeled RBCs flowing near the microchannel wall $(z=20 \mu \mathrm{m})$. 


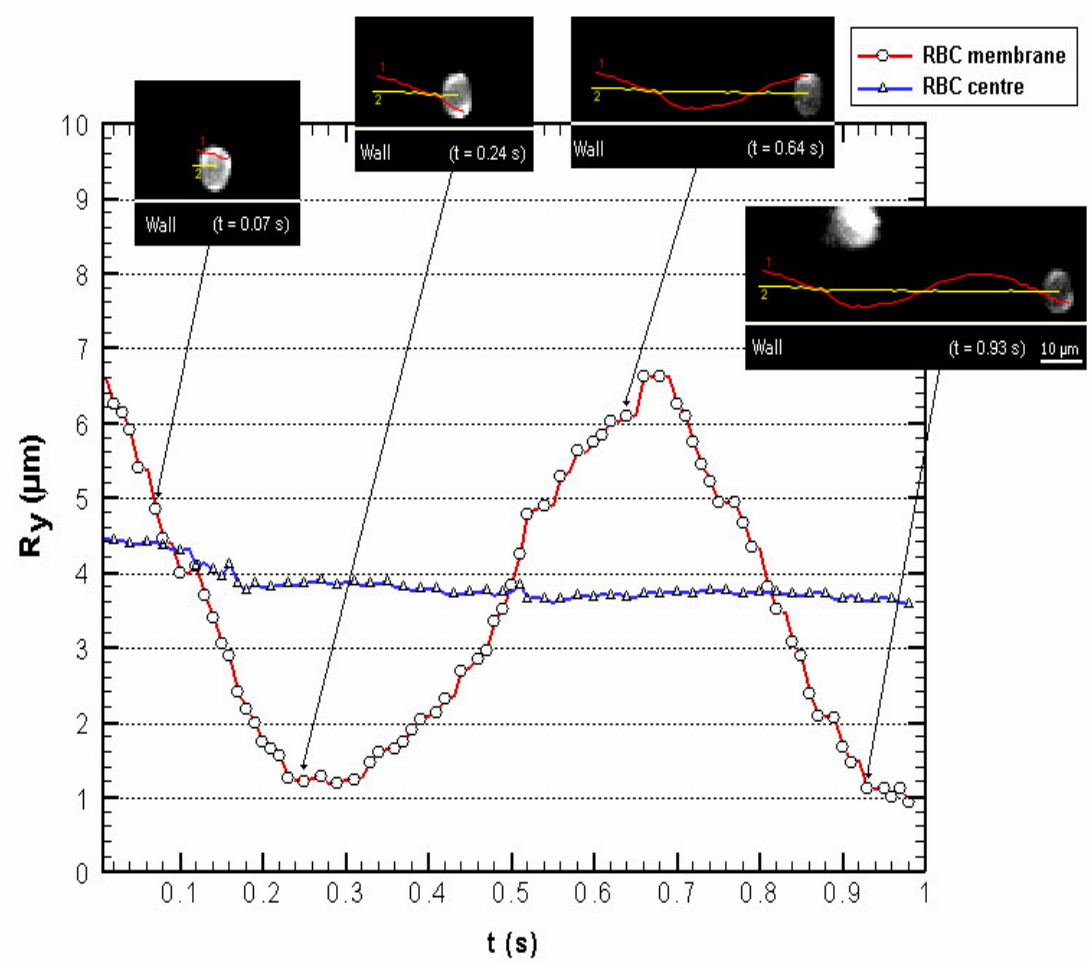

a)

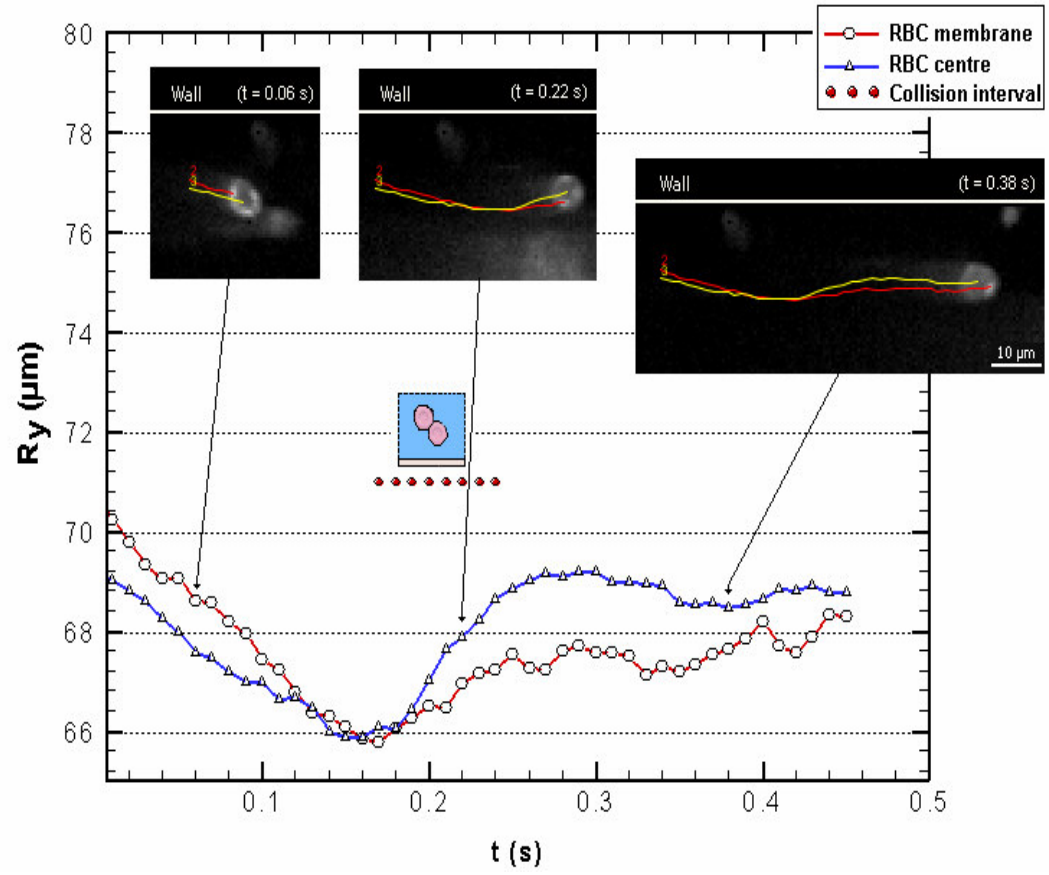

b)

Figure 9. Instantaneous transversal displacement $\left(\mathrm{R}_{\mathrm{y}}\right)$ of translational and rotational motion $(\operatorname{Re} \sim 0.006)$ at 10 $\mathrm{ms}$ time intervals tracked in the bottom plane $(z=20 \mu \mathrm{m})$ of a $100 \mu \mathrm{m}$ diameter microchannel a) without interaction and $b$ ) with interaction. The RBC rolling along the wall, which does not suffer any collision, tends to rotate in a regular and periodic way. In contrast, the RBC interacting with neighboring cells rotates in an erratic manner. See Supplementary Video 4 and 5. 
The RBC without interaction was measured along the wall surface. This RBC did not interact with a neighboring $\mathrm{RBC}$, allowing its translational transversal displacement to remain almost constant. Moreover, the RBC rotated as a biconcave disk in a clockwise direction with periodically varying angular motion. In contrast, RBCs interacting with neighboring cells demonstrated changes in both translational and rotational motions. These RBCs rotated in an erratic manner rather than in a regular and periodic way.

\section{$R B C-W B C$ interactions in flowing blood}

The hemodynamic interaction effects of WBCs on the motion of RBCs were also measured. Figure 10 shows the paths and corresponding transversal displacements of a RBC (RBC 5) interacting with the center upper part of a WBC (upper) and a RBC (RBC 6) interacting with the left side of the WBC (lower). Note that RBC 5 is located in the focal plane (high intensity), while the WBC is below the focal plane (lower intensity). Even though the WBC is not in the focal plane, the motion of RBCs is disturbed by its presence in the flow because of its size (typically twice that of a RBC) and geometry. Moreover, for both cases, the transversal displacement increased when a collision with a WBC occurred (Fig. 10). However, the displacement seemed to increase considerably when the RBC interacted with the side of a WBC. According to our observations, when RBCs interact around the center of a WBC, the displacement is larger in the $z$ direction (depth of the microchannel). Although it was possible to visualize this three-dimensional displacement, our system does not presently allow quantification.

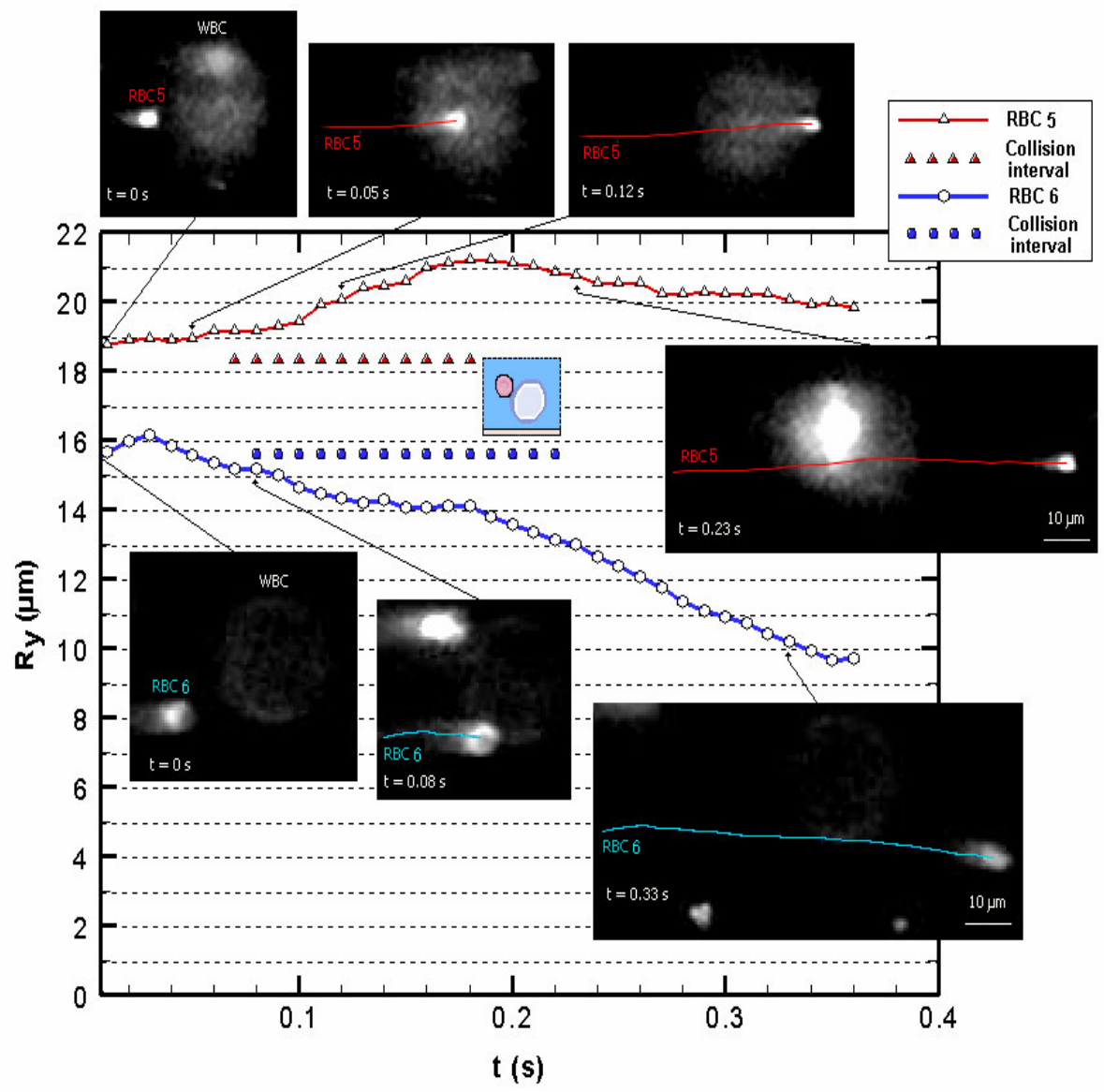

Figure 10. RBC-WBC interactions at $10 \mathrm{~ms}$ time intervals tracked in the bottom plane $(z=20 \mu \mathrm{m})$ of a $100 \mu \mathrm{m}$ diameter microchannel ( $\mathrm{Re} \sim 0.006)$. Instantaneous transversal displacement $\left(\mathrm{R}_{\mathrm{y}}\right)$ of $\mathrm{RBC} 7$ interacting with center upper part of a WBC (upper) and RBC 8 interacting with the left side of the WBC (lower). The RBCs paths are diverted due to collisions with WBCs however the lateral displacement seems to increase when the RBC interacts with the WBC lateral surface. 


\section{Dispersion of labeled RBCs at different Hets}

The random transversal motions of RBCs in directions normal to the flow can be analyzed using a dispersion coefficient $\left(D_{y y}\right)^{16,33}$ :

$D_{y y}(t)=\frac{1}{N} \sum_{i=1}^{N} \frac{\left\langle\left(R_{i, y}(t)-R_{i, y}(0)\right)^{2}\right\rangle}{2 t}$,

where $\left\langle\left(R_{i, y}(t)-R_{i, y}(0)\right)^{2}\right\rangle, t$, and $N$ are the mean square displacement, time interval, and number of measured RBCs, respectively. Note that $R_{i, y}(t)-R_{i, y}(0)$ is the traversal displacement of individual $\mathrm{RBC} i$ in the $x y$ confocal plane over some time interval, $t$.

The paths of multiple labeled RBCs were measured in the center plane and in focal planes located at $35 \mu \mathrm{m}$ and $20 \mu \mathrm{m}$ from the bottom of the microchannel (see Table 2). Figure 11 shows typical transversal displacements of RBCs in different focal planes for two different concentrations of RBCs (15 and 35\% Hct). The corresponding RBC dispersion coefficients $\left(D_{y y}\right)$ in three different focal planes for each Hct are shown in Figure 12. The results show that $D_{y y}$ tends to increase moving away from the microchannel axis, suggesting that the velocity gradients induced by the wall have a strong influence on the erratic transversal displacements shown in Figure 12.

Table 2. Results of dispersion of labeled RBCs at different in-focus planes for $15 \%$ and $35 \%$ Hct.

\begin{tabular}{cccc}
\hline Experiment & $\mathrm{n} *$ & $\begin{array}{c}\text { In-focus plane } \\
(\mathrm{z} \text {-axis, } \mu \mathrm{m})\end{array}$ & $\begin{array}{c}D_{y y}{ }^{\S} \pm \mathrm{SD} \\
\left(\mathrm{cm}^{2} / \mathrm{s}\right)\end{array}$ \\
\hline $\begin{array}{c}\text { RBC dispersion for } \\
\text { 15\% Hct }\end{array}$ & 70 & 50 & $3.0 \times 10^{-8} \pm 6.4 \times 10^{-9}$ \\
& 75 & 35 & $4.2 \times 10^{-8} \pm 7.0 \times 10^{-9}$ \\
& 78 & 20 & $4.2 \times 10^{-8} \pm 6.4 \times 10^{-9}$ \\
RBC dispersion for & 53 & 50 & $4.3 \times 10^{-8} \pm 1.0 \times 10^{-8}$ \\
35\% Hct & 60 & 35 & $5.3 \times 10^{-8} \pm 1.2 \times 10^{-8}$ \\
& 70 & 20 & $6.5 \times 10^{-8} \pm 1.1 \times 10^{-8}$ \\
\hline
\end{tabular}

*Number of labeled RBCs.

${ }^{\S}$ Last value from the diagrams presented in Figure 12. 

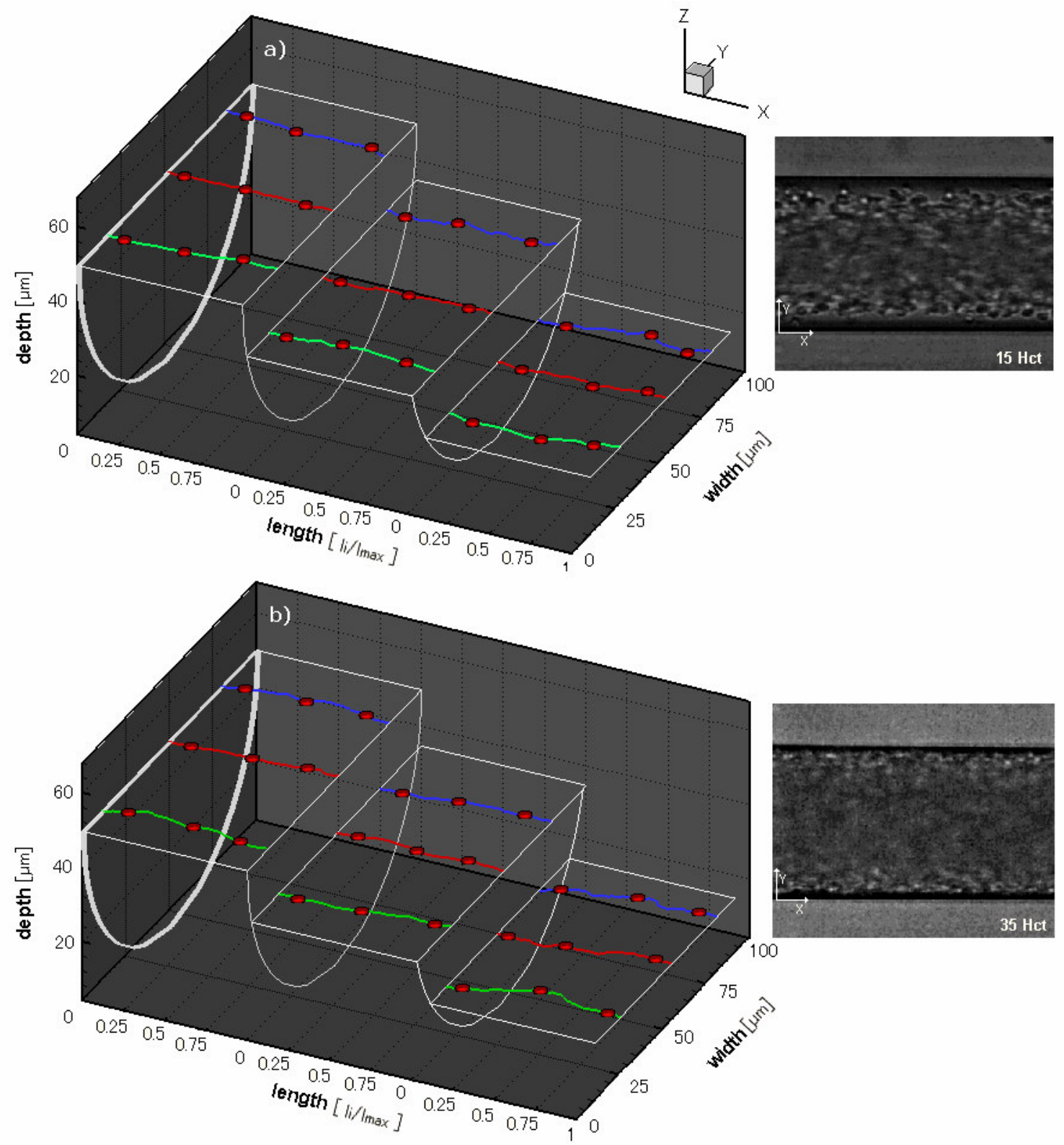

Figure 11. Instantaneous transversal displacement $\left(\mathrm{R}_{\mathrm{y}}\right)$ of labeled RBCs at different in-focus planes for a) $15 \%$ Hct and b) $35 \%$ Hct $(\mathrm{Re} \sim 0.004)$. The RBC positions normal to the direction of the flow tend to increase moving away from the centre plane of the microtube. 


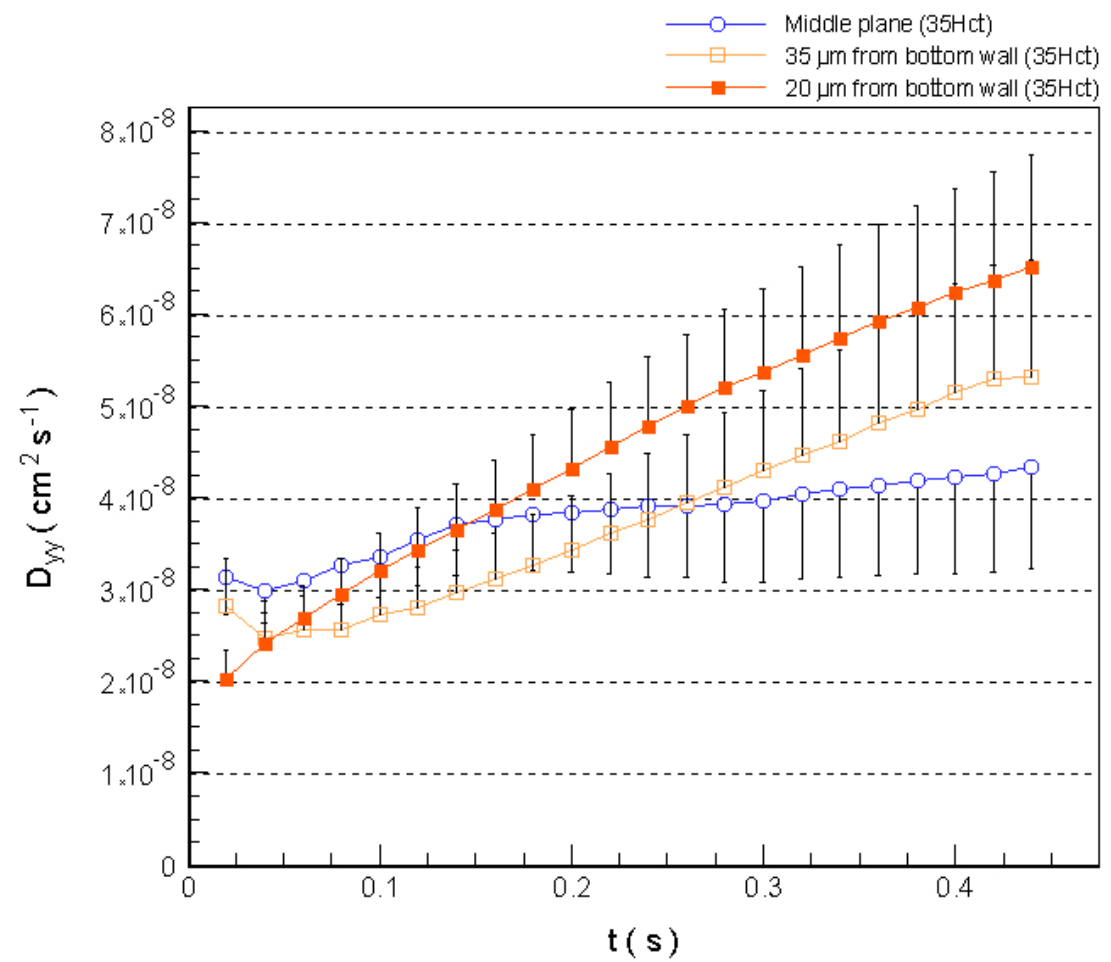

a)

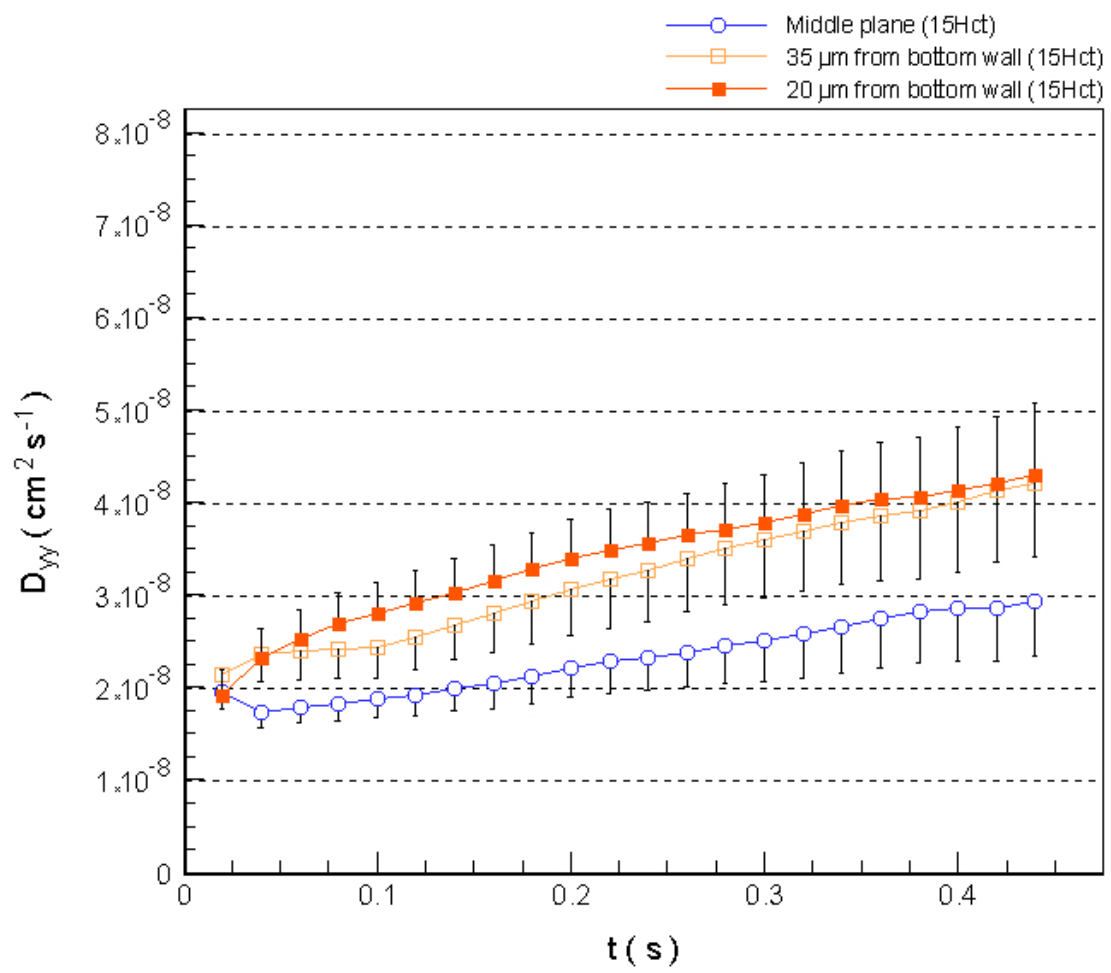

b)

Figure 12. RBC transversal dispersion coefficients $\left(D_{y y}\right)$ at different in-focus planes for a) $15 \%$ Hct and b) $35 \%$ Hct $(\operatorname{Re} \sim 0.004) . D_{y y}$ tends to increase moving away from the centre plane of the microtube. This phenomenon is a consequence of the combination of both radial and circumferential effects on the RBCs motions. Measured values are expressed as the mean \pm standard deviation (95\% confidence interval). 


\section{DISCUSSION}

Past research has identified temporal fluctuations in blood velocity profiles in microvessels. Most of these findings resulted from studies performed at macroscale and mesoscale levels. We believe that it is crucial to investigate the cause of this phenomenon at a cellular level. The data presented in this study demonstrate the ability of the confocal micro-PTV system to provide further insight into the complex flow behavior in microcirculation.

\section{Blood cell interactions in flowing blood}

Limitations in the current optical techniques that are used to investigate blood flow at a microscopic level have led to numerical studies on cell-cell interactions ${ }^{34,35}$. Nevertheless, experimental studies are essential to validate these numerical predictions. The present study quantitatively and qualitatively characterized cell-cell hemodynamic interactions in flowing blood. The Re used in this study ranged from 0.0038 to 0.0058 . At these low Re values, we did not observe obvious RBC aggregation. The use of Dextran 40 might also have reduced the tendency toward aggregation.

The hemodynamic interactions of RBCs in concentrated suspensions depend on multi-physics hemodynamic parameters such as shear rate, plasma layer, and RBC deformability. We presented two typical RBC-RBC interactions in flowing blood. Generally, upon RBC interaction, transversal displacement tends to increase to a maximum peak. The cell rarely returns to its initial position, as interactions with other cells are likely. Two interesting phenomena happening within the plasma layer are presented. First, around the boundary of the plasma layer, where cells are crowded, migration toward the middle was inverted, owing to a hemodynamic interaction with a RBC flowing in the crowded region (Fig. 6). Second, when a RBC was rolling on the surface, an interaction did not cause a significant increase in transversal displacement, indicating that the lubrication force between the RBC and the wall is greater than the hemodynamic force caused by the interaction (Fig. 7). Our results indicate that the plasma layer formed in microchannels is not completely cell-free. Furthermore, these results suggest that a RBC flowing in the plasma layer is subjected to both axial and lateral migration, generating an undulating trajectory. Moreover, multi-body collisions continuously divert RBC trajectories, inducing flow disturbances at a microscopic level. A statistical analysis based on a large number of collisions can be found in a recent study performed by the current authors ${ }^{37}$.

Generally, in dilute suspensions (Hct, $\sim 3 \%$ ), RBCs tended to follow linear trajectories, as collisions with a neighboring cell were unlikely to occur. As the Hct increased, the RBCs began to experience transversal displacements of about $2 \%$ from the original path. At moderate Hcts $(\sim 20 \%)$ the RBCs flowing within the plasma layer tended to migrate toward the axis; however this tendency was opposed in crowded region. Such resistance to crowding can enhance the fluctuations of the RBC trajectories, which eventually lead to an increase in wall collisions. This phenomenon may play an important role in the blood mass transport of cells and proteins to tissues and thrombi.

During our experiments, most of the WBCs were flowing near the bottom wall of the microtube. In the present study, two representative RBC-WBC interactions were analyzed (Fig. 10). Note that some measurements of cell paths were performed with faint images (slightly out-of-focus cells). Our results suggest that the WBC hemodynamic effect on the trajectory of a $\mathrm{RBC}$ is dependent on the contact angle between the cells. For example, the transversal displacement of RBC 5 increased by about $2 \mu \mathrm{m}$ (Fig. 10) after it interacted with the upper center periphery of the WBC, while the transversal displacement of RBC 6 increased dramatically $(>4 \mu \mathrm{m})$ after it interacted with the lateral surface of the WBC.

\section{Orientation of RBCs in flowing blood}

In our experiments (Re, 0.0071), we did not observe marked deformation, especially around the plasma layer. However, the degree of deformation seemed to increase as the RBCs migrated toward 
the center of the microchannel. At this region of high concentration, RBCs tended to get squeezed as they passed each other. Our study presents two remarkable examples of RBC orientation at low Re. The proposed technique provides information about both translational and rotational motions of a RBC with and without interaction. As shown in Figure 9, interacting RBCs rotated erratically compared with cells rolling along the wall without interactions.

\section{RBC dispersion coefficient}

The present study provides quantitative data on the transversal fluctuations of RBCs in different focal planes and at different Hcts. The transversal displacement and corresponding transversal dispersion coefficient tended to increase with decreasing $\mathrm{z}$ position (see Fig. 11, 12). Thus, the RBCs that flowed near the wall had the highest transversal dispersion, which was a consequence of the combination of both radial and circumferential effects on the motions of RBCs traveling in the planes oriented out from the center axis. These results reinforce the evidence that both the local Hct and the velocity gradients induced by the wall play important roles in blood flow properties, which influence the resistance to blood flow in microvessels. Our detailed investigation of the complex effect of the Hct and microchannel diameter on the RBC radial dispersion coefficient can be found in our previous report, Lima et al., $2008^{37}$.

In previous studies, Goldsmith and colleagues ${ }^{4,5,16,18}$ calculated radial dispersion coefficients using a fluid containing $39 \%$ ghost cells. Their values were slightly higher than the present results, especially near the wall ${ }^{37}$. This discrepancy might have been due to their use of RBCs without hemoglobin, which would affect RBC rheological properties. Moreover, the out-of-focus effect might have contributed to the measurements on RBCs located away from the focal plane of interest. A more detailed comparison can be found in Lima et al., $2008^{37}$.

\section{Main advantages and limitations of the proposed confocal micro-PTV}

One of the main advantages of the proposed confocal micro-PTV system is the ability of the spinning disk confocal microscope (SDCM) to minimize background noise from the out-of-focus planes. Using a conventional microscope, the intensity of the RBCs in the out-of-focus planes does not change with the distance from the focal plane, resulting in an estimated depth of field ${ }^{38}$ of up to four times the size of RBCs. In contrast, the brightness of the RBCs imaged with SDCM decreases dramatically near (1 $\mu \mathrm{m}$ from) the focal plane, providing greater contrast and true thin optical sections ( $2 \mu \mathrm{m}$ in the current study). Hence, SDCM systems provide accurate measurements of the paths of single RBCs or multi$\mathrm{RBC}$ interactions flowing in the same focal plane, which is not possible using a conventional microscope.

One of the main limitations of using the SCDM is the low blood flow rate required to obtain accurate measurements. Our confocal system uses a CSU-22 scanning unit from Yokogawa, which allows us to obtain images at a rate of up to 2,000 frames/s ( $0.5 \mathrm{~ms}$ between a pair of images). Although this temporal resolution can be achieved using dilute suspensions of RBCs, the images recorded using concentrated suspensions were too dark to be processed. Reasons for this limitation include not only the loss of incident illumination induced by the spinning disk pinholes but also the effect of the light scattered and absorbed by the RBCs. However, by decreasing the temporal resolution to 100 frames/s, it was possible to use a long exposure time $(9.4 \mathrm{~ms})$; this improved the image brightness, because the increase in the residence time of the labeled RBCs allowed the collection of sufficient photons for image formation. This approach was adequate for successfully measuring velocities up to $0.52 \mathrm{~mm} / \mathrm{s}$ $(\mathrm{Re}, \sim 0.007)$ in the center plane of the microchannel ${ }^{26}$. As the velocities in the microcirculation are typically between 0.2 and $10 \mathrm{~mm} / \mathrm{s}^{17}$, our approach is suitable for studying microcirculation phenomena, especially in capillaries where the velocities are usually less than $1 \mathrm{~mm} / \mathrm{s}$.

The primary aim of the present study was to develop a protocol for labeling RBCs with enough fluorescent probe to obtain images of sufficient quality for measurements using our confocal system. According to previous experiments ${ }^{16}$, healthy RBCs exhibit appreciable migration, whereas hardened RBCs do not. Recent experiments performed in a stenosed microchannel ${ }^{39}$ have also shown clear differences in migration between healthy and hardened cells. These studies suggest that cell migration 
is strongly affected by cells deformability. Although in our experiments we always observed flows with a cell-free plasma layer on the wall and a cell-rich center core, we did not study the influence of the labeling procedure on the deformability of RBCs. In the near future, we expect to measure quantitatively the mechanical properties of individual labeled RBCs in order to determine the best experimental protocol, taking into account the compromise between the intensity of the emitted light versus the deformability of RBCs.

\section{CONCLUSIONS}

We applied a confocal micro-PTV system to measure the motion of individual RBCs through a 100$\mu \mathrm{m}$ microchannel. The fluorescently labeled RBCs exhibited flow characteristics similar to those reported for flows in microvessels in vivo. The present study characterized cell-cell hemodynamic interactions, $\mathrm{RBC}$ orientation, and $\mathrm{RBC}$ transversal dispersion at different depths in flowing blood with various hematocrit levels. In general, the results indicate that the plasma layer enhances collisions between neighboring RBCs, thereby influencing the motion and flow behavior of the cells. The results also provide evidence that multi-body collisions promote fluctuations in the translational and rotational motions of RBCs. These findings confirm earlier observations and provide new insights into the complex behavior of RBCs flowing in a crowded environment. In summary, we have provided evidence that the proposed confocal micro-PTV system is a powerful technique for monitoring the paths of two or more blood cells interacting in the same focal plane. This method eliminates some difficulties with previous methods and provides additional details on the motions of RBCs that have not been obtainable with conventional methods.

\section{ACKNOWLEDGEMENTS}

This study was supported in part by the following grants: International Doctoral Program in Engineering from the Ministry of Education, Culture, Sports, Science and Technology of Japan (MEXT), "Revolutionary Simulation Software (RSS21)" next-generation IT program of MEXT; Grants-in-Aid for Scientific Research from MEXT and JSPS Scientific Research in Priority Areas (768) "Biomechanics at Micro- and Nanoscale Levels", "Scientific Research (S) No.19100008".

\section{REFERENCES}

1. Fahraeus, R., Lindqvist, T., The viscosity of the blood in narrow capillary tubes. Am. J. Physiol. 96: 562-568, 1931.

2. Schmid-Schonbein, H., Wells, R., Fluid drop-like transition of erythrocytes under shear. Science 165: 288-291, 1969.

3. Chien, S., Shear dependence of effective cell volume as a determinant of blood viscosity. Science 168: 977979, 1970.

4. Goldsmith H., Red cell motions and wall interactions in tube flow, Federation Proceedings 30: 1578-1588, 1971.

5. Goldsmith H., Deformation of human red cells in tube flow, Biorheology 7: 235-242, 1971.

6. Fischer T., Stohr-Lissen M, Schmid-Schonbein, H., The red cell as a fluid droplet: tank tread-like motion of the human erythrocyte membrane in shear flow. Science 202: 894-896, 1978.

7. Gaehtgens P., Meiselman H., Wayland H., Velocity profiles of human blood at normal and reduced hematocrit in glass tubes up to $130 \mu$ diameter. Microvascular Research 2: 13-23, 1970.

8. Baker M., Wayland H., On-line volume flow rate and velocity profile measurement for blood in microvessels. Microvascular Research 7: 131-143, 1974.

9. Parthasarathi A., Japee S., Pittman R., Determination of red blood cell velocity by video shuttering and image analysis. Annals of Biomedical Engineering 27: 313-325, 1999.

10. Born G., Melling A., Whitelaw J., Laser Doppler microscope for blood velocity measurement. Biorheology 15: 163-172, 1978.

11. Uijttewaal W., Nijhof E., Heethaar R., Lateral migration of blood cells and microspheres in two-dimensional Poiseuille flow: a laser Doppler study. Journal of Biomechanics 27: 35-42, 1994.

12. Vennemann P., K. Kiger, R. Lindken, B. Groenendijk, S. Stekelenburg-de Vos, T. Hagen, N. Ursem, R. Poelmann, J. Westerweel, B. Hierk, In vivo micro particle image velocimetry measurements of bloodplasma in the embryonic avian heart. Journal of Biomechanics 39: 1191-1200, 2006. 
13. Sugii Y, Okuda R, Okamoto K, Madarame H, Velocity measurement of both red blood cells and plasma of in vitro blood flow using high-speed micro PIV technique. Measurement Science and Technology 16: 11261130, 2005.

14. Lima, R., Wada, S., Takeda, M., Tsubota, K., Yamaguchi, T., In vitro confocal micro-PIV measurements of blood flow in a square microchannel: the effect of the haematocrit on instantaneous velocity profiles. Journal of Biomechanics 40: 2752-2757, 2007.

15. Lima, R., Wada, S., Tanaka, S., Takeda, M., Ishikawa, T., Tsubota, K., Imai, Y., Yamaguchi, T., In vitro blood flow in a rectangular PDMS microchannel: experimental observations using a confocal micro-PIV system. Biomedical Microdevices, 10: 153-167, 2008.

16. Goldsmith, H., Turitto, V., Rheological aspects of thrombosis and haemostasis: basic principles and applications. ICTH-Report-Subcommittee on Rheology of the International Committee on Thrombosis and Haemostasis. Thromb Haemost. 55: 415-435, 1986.

17. Caro, C., Pedley, T., Schroter, R., Seed W., The mechanics of the circulation, Oxford University Press, 1978.

18. Goldsmith, H., Marlow, J., Flow behavior of erythrocytes. II. Particles motions in concentrated suspensions of ghost cells, Journal of Colloid and Interface Science 71: 383-407, 1979.

19. Nash, G., Meiselman, H., Red cell and ghost viscoelasticity. Effects of hemoglobin concentration and in vivo aging. Biophysical Journal 43: 63-73, 1983.

20. Shiga, T., Maeda N., Kon K., Erythrocyte rheology. Crit. Rev. Oncol. Hematol. 10: 9-48, 1990.

21. Meinhart C, Wereley S, Santiago J., PIV measurements of a microchannel flow. Experiments in Fluids 27: 414-419, 1999.

22. Conchello J., Lichtman, J., Optical sectioning microscopy. Nature Methods 2: 920 - 931, 2005.

23. Inoue, S., Inoue, T., Direct-view high-speed confocal scanner: the CSU-10. Cell Biological Applications of Confocal Microscopy, Academic Press, 87-127, 2002.

24. Tanaani T, Otsuki S, Tomosada N, Kosugi Y, Shimizu M. Ishida H., High-speed 1-frame/ms scanning confocal microscope with a microlens and Nipkow disks. Applied Optics 41: 4704-4708 2002.

25. Park J, Choi C, and Kihm K, Optically sliced micro-PIV using confocal laser scanning microscopy (CLSM). Experiments in Fluids 37: 105-119, 2004.

26 Lima, R., Wada, S., Tsubota, K.,Yamaguchi, T., Confocal micro-PIV measurements of three dimensional profiles of cell suspension flow in a square microchannel. Measurement Science and Technology 17: 797808, 2006.

27. Kinoshita, H., Kaneda, S., Fujii, T.,Oshima, M., Three-dimensional measurement and visualization of internal flow of a moving droplet using confocal micro-PIV. Lab Chip 7: 338-346, 2007.

28. Abramoff, M., Magelhaes, P., Ram, S., Image Processing with Image J, Biophotonics International 11: 36-42, 2004.

29. Meijering E., Smal I., Danuser G., Tracking in Molecular Bioimaging, IEEE Signal Processing Magazine, 23: 46-53, 2006.

30. Lima, R., Analysis of the blood flow behavior through microchannels by a confocal micro-PIV/PTV system. Ph.D. Thesis, Tohoku University, Japan, 2007.

31. Wootton, D., Ku D., Fluid mechanics of vascular systems, diseases, and thrombosis. Annual Review of Biomedical Engineering 1: 299-329, 1999.

32. Miyazaki, H. and Yamaguchi, T., Formation and destruction of primary thrombi under the influence of blood flow and von willebrand factor analysed by a D. E. M., Biorheology 40: 265-272, 2003.

33. Ishikawa, T. and Pedley, T., Diffusion of swimming model micro-organisms in a semi-dilute suspensions. Journal of Fluid Mechanics, 588: 437-462, 2007.

34. Tsubota, K., Wada, S., Yamaguchi, T., Particle method for computer simulation of red blood cell motion in blood flow. Computer Methods and Programs in Biomedicine 83: 139-146, 2006.

35. Yamaguchi, T., Ishikawa, T., Tsubota, K., Imai, Y., Nakamura M., Fukui T., Computational blood flow analysis - new trends and methods. Journal of Biomechanical Science and Enginneering 1: 29-50, 2006.

36. Munn, L., Melder R., Jain, R., Role of erythrocytes in leukocyte-endothelial interactions: mathematical model and experimental validation. Biophysical Journal 71: 466-478, 1996.

37. Lima, R., Ishikawa T., Imai, Y., Takeda, M., Wada, S., Yamaguchi, T., Radial dispersion of red blood cells in blood flowing through glass capillaries: role of hematocrit and geometry. Journal of Biomechanics 41: 2188-2196, 2008.

38. Meinhart C, Wereley S., Gray H., Volume illumination for two-dimensional particle image velocimetry. Measurement Science and Technology 11: 809-814, 2000.

39. Fujiwara, H., Ishikawa T., Lima, R., Matsuki, N., Imai, Y., Kaji, H., Nishizawa, M., Yamaguchi, T., Red blood cell motions in high-hematocrit blood flowing through a stenosed microchannel. Journal of Biomechanics , 42: 838-843, 2009. 